LAWRENCE LIVERMORE N A T IO N A L LABORATORY
Radiation Detection Field Test at the Federal Express (FedEx) Air Cargo Facility at Denver International Airport (DIA)

A. Waters, D. Weirup, H. Hall, A. Dougan, D. Trombino, G. Mattesich, E. Hull, S. Bahowick, A. Loshak, J. Gruidl

February 24, 2004

SPIE - Smart Structures/NDE 2004

San Diego, CA, United States

March 14, 2004 through March 18, 2004 
This document was prepared as an account of work sponsored by an agency of the United States Government. Neither the United States Government nor the University of California nor any of their employees, makes any warranty, express or implied, or assumes any legal liability or responsibility for the accuracy, completeness, or usefulness of any information, apparatus, product, or process disclosed, or represents that its use would not infringe privately owned rights. Reference herein to any specific commercial product, process, or service by trade name, trademark, manufacturer, or otherwise, does not necessarily constitute or imply its endorsement, recommendation, or favoring by the United States Government or the University of California. The views and opinions of authors expressed herein do not necessarily state or reflect those of the United States Government or the University of California, and shall not be used for advertising or product endorsement purposes. 


\title{
Radiation Detection Field Test at the Federal Express (FedEx) Air Cargo Facility at Denver International Airport (DIA) ${ }^{1}$
}

\author{
Amy Waters, Dave Weirup, Howard Hall, Arden Dougan, Dave Trombino, Gary \\ Mattesich, Ethan Hull, Sally Bahowick, Alex Loshak, and Jeremiah Gruidl \\ Nonproliferation, Arms Control, and International Security Directorate \\ Lawrence Livermore National Laboratory
}

\section{Executive Summary}

Lawrence Livermore National Laboratory (LLNL) recently conducted a field-test of radiation detection and identification equipment at the air cargo facility of Federal Express (FedEx) located at Denver International Airport (DIA) over a period of two weeks. Comprehensive background measurements were performed and were analyzed, and a trial strategy for detection and identification of parcels displaying radioactivity was implemented to aid in future development of a comprehensive protection plan. The purpose of this project was threefold:

- Quantify background radiation environments at an air cargo facility.

- Quantify and identify "nuisance" alarms.

- Evaluate the performance of various isotope identifiers deployed in an operational environment (in this case, the operational environment included the biggest blizzard in over 90 years!).

LLNL emplaced a primary screening detector that provided the initial detection of radiation anomalies in near real-time. Once detected, a secondary test location provided capability to perform higher-resolution analysis of the parcels or containers that triggered the primary detector. The primary detector used for this project was a LLNL developed prototype Adaptable Radiation Area Monitor (ARAM). The ARAM consists of a large $\mathrm{NaI}(\mathrm{Tl})$ scintillating gamma-ray detector, self-contained with a computer for data storage. The secondary screening detectors consisted of three commercial off the shelf (COTS) handheld isotope identifiers, and one $140 \%$ efficiency cryogenically cooled High-Purity Germanium (HPGe) detector.

The ARAM detector was positioned for 3 evening shifts directly underneath a conveyor belt to screen individual packages. The detector screened over 24,000 individual packages with zero detected events as defined above. The ARAM detector was then positioned to screen larger Unit Loading Devices (ULDs) as they were unloaded from trucks for 4 evening shifts. Approximately 28 trucks were unloaded and screened and two triggered radiation events were observed. Both of the radiation events were determined to be legitimate shipments of radioactive material. The radioactive materials were identified as ${ }^{131} \mathrm{I}$ and ${ }^{123} \mathrm{I}$, and the packages were clearly labeled as "excepted package - limited quantity" and Radioactive Class II, respectively. Both of these materials are routinely used in nuclear medicine. Performance of the handheld units

\footnotetext{
${ }^{1}$ This work was performed under the auspices of the U. S. Department of Energy by the University of California Lawrence Livermore National Laboratory under contract No. W-7405-Eng-48.
} 
varied, and often the identifications were not consistent. In all cases, the HPGe was used to confirm isotope identity.

The two primary locations selected for the ARAM resulted in little impact to FedEx operations. When the radiation events were detected, handheld units were used to quickly search and locate the packages emitting radiation as they were unloaded from the larger ULD by FedEx personnel. The overall effect on FedEx operations and personnel was deemed to be minimal. The staff of FedEx, both in Denver and at FedEx headquarters in Memphis, were extremely supportive and easy to work with and seemed pleased with the performance of the ARAM and the handheld units.

\section{Introduction}

Currently there is significant interest in finding ways to limit the vulnerability of commercial shipping routes that could be used as the pathway for delivery of a terrorist weapon of mass destruction (WMD) or disruption to an intended target. A nuclear weapon, materials to make a nuclear device, or a radiological dispersal device (RDD) delivered via normal US commerce would result in massive disruption or casualties. U.S. airlines transport billions of tons of cargo each year by air, in both passenger and allcargo planes. Typically one half of the hull of a passenger plane is filled with cargo [1].

For this project, an air cargo facility was selected to partner with LLNL for a two-week field test to investigate the radiation environment at such facilities, to quantify and identify nuisance alarms and to evaluate detectors in an operational environment. Passive radiation detectors were used to measure background radiation over several days as well as to screen cargo shipped through the Federal Express (FedEx) facility in Denver (see Figure 1) to help determine the feasibility of deploying such detectors for use as one security screening mechanism. Primarily outgoing cargo was screened in an attempt to comply with suggestions from US Customs for air carriers to screen cargo at the point of origin, or offshore. In other words, if a WMD arrives in the U.S. on an aircraft, it may be too late for interdiction and disablement of the device.

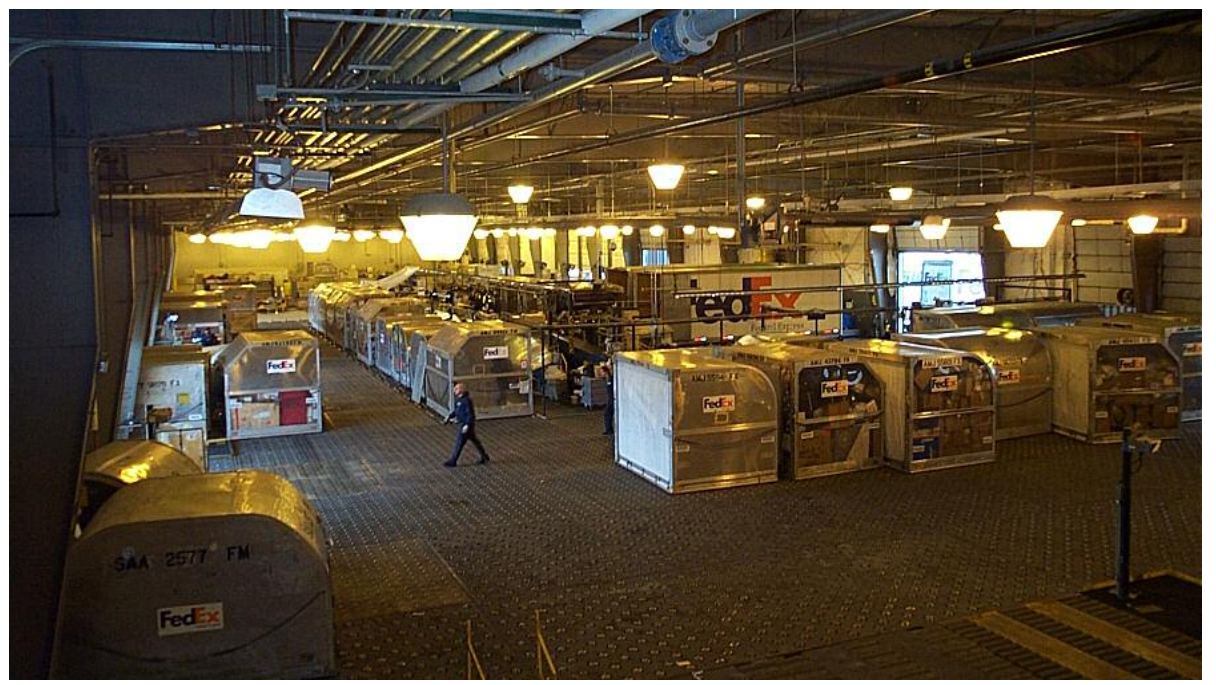

Figure 1. The Federal Express Air Cargo facility at Denver International Airport. 


\section{Instrumentation and Techniques}

\section{Primary Detector}

The technique chosen for this field test was to emplace a robust primary detector for initial radiation event triggering, with more sensitive secondary equipment used for confirmation as shown in Figure 2.

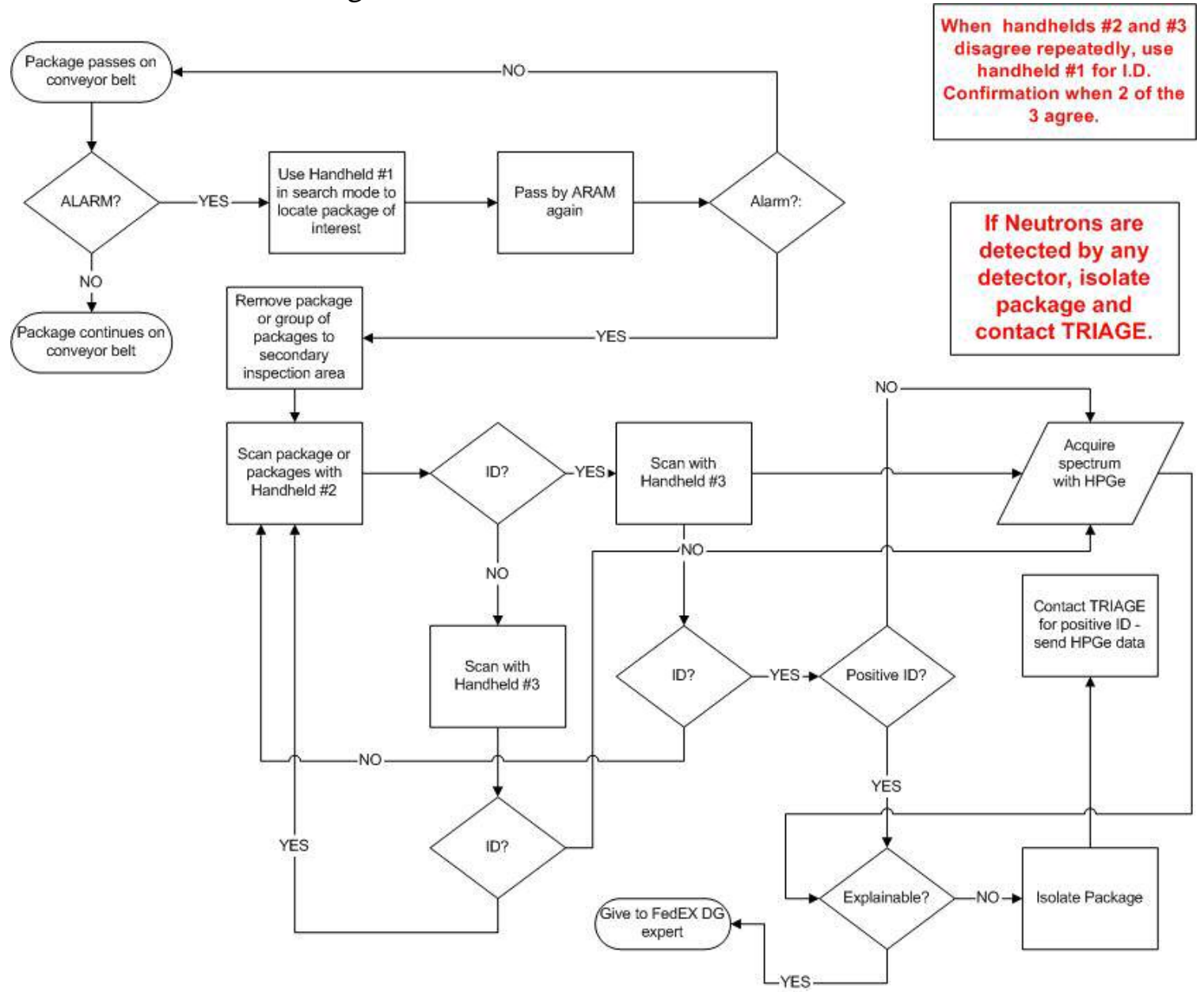

Figure 2. Procedural flow chart used for the radiation detection field test at the FedEx Air Cargo facility at DIA. "Triage" is a DOE program developed to support first responders.

The primary detector used in this experiment was a LLNL developed stand-alone system called the Adaptive Radiation Area Monitor (ARAM). The ARAM was developed to detect small quantities of moving radioactive sources. It consists of a large Thallium doped Sodium Iodide ( $\mathrm{NaI}(\mathrm{Tl})$ ) scintillator coupled to an Ortec microBASE 1024 channel universal multichannel analyzer (MCA). The ARAM detector, computer and associated electronics are packaged in a ruggedized case with overall dimensions of $3 \times 2 \times 2 \mathrm{ft}^{3}$ and a total weight of $200 \mathrm{lbs}$. The ARAM uses LLNL developed software to collect scalar and spectral data, that can be optimized for both detection and identification probability. Data collected by the ARAM internal computer can be displayed through an Ethernet 
connection on a connected laptop computer screen as a streaming chart of total gross counts, and spectra. When a defined radiation event occurs, the scrolling gross counts window on the laptop turns red and audiblyalerts the operator that an event has occurred. Data files were retrieved after each shift for more detailed analysis.

\section{Secondary Detectors}

Three commercially available handheld isotope identifiers were used to confirm and identify radiation sources after an event. The secondary handheld detectors used in this experiment were the SAM 935 manufactured by Berkeley Nucleonics, the fieldSpec-He3 by Thermo RMP, and the GR-130 by Exploranium. Each of the handheld detectors uses $\mathrm{NaI}(\mathrm{Tl})$ crystals for gamma-ray detection. The SAM 935 employs a $1.5 \times 2$ inch $^{2} \mathrm{NaI}(\mathrm{Tl})$ crystal for detection of gamma rays as well as a $20 \mathrm{~atm}^{3} \mathrm{He}$ tube for detection of neutrons. A small ${ }^{137} \mathrm{Cs}$ source is attached to the unit for calibration prior to use. The fieldSpec employs a $1 \times 2$ inch $^{2} \mathrm{NaI}(\mathrm{Tl})$ crystal and a small ${ }^{3} \mathrm{He}$ tube, and has a built in ${ }^{137} \mathrm{Cs}$ source for calibration. The Exploranium GR-130 employs a $1.5 \times 2.2 \mathrm{inch}^{2} \mathrm{NaI}(\mathrm{Tl})$ crystal and does not have neutron detection capability. $\mathrm{A}^{137} \mathrm{Cs}$ source is supplied with the unit for calibration purposes.

In addition to the handheld secondary detectors, a cryogenically cooled, $140 \%$ efficiency HPGe detector was also used. Data acquired with the HPGe detector was analyzed by a trained spectroscopist to confirm identifications made with the handheld detectors. The HPGe was also used to acquire background radiation measurements over 48 hours.

\section{Operations}

The ARAM was located for 3 evening shifts underneath the conveyor belt (see Figure 3) to screen individual packages (the original plan to acquire data for 5 shifts was modified due to severe weather and the closure of DIA). To be sorted on the conveyor belt, packages weighed less than 150 pounds, with dimensions less than $3 \mathrm{ft}$ on a side.

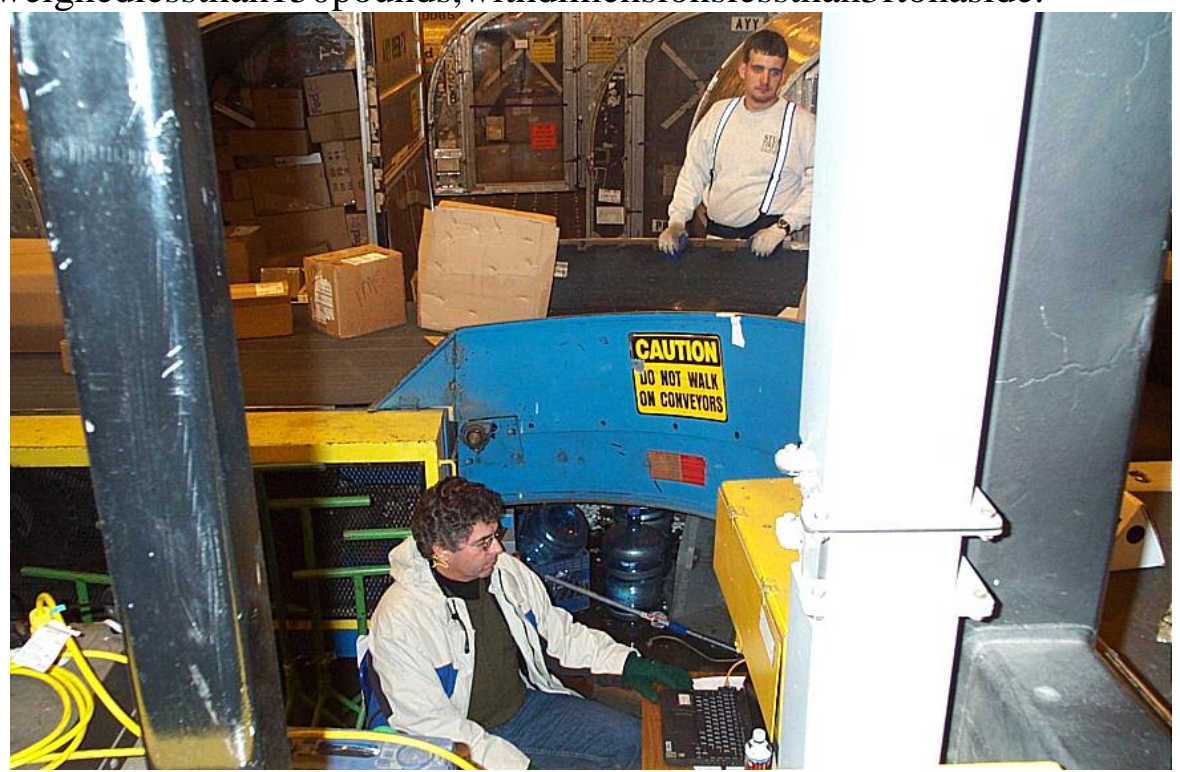

Figure 3. Conveyor belt at the Denver FedEx facility. The ARAM is located directly underneath the curved part of the belt. 
The ARAM was also located for 4 evening shifts between two truck doors to screen large Unit Loading Devices (ULDs) as they were offloaded from trucks. Several loaded ULDs are shown in Figure 4. Additionally, continuous background radiation measurements were acquired over two consecutive weekends with the HPGe detector followed by the ARAM.

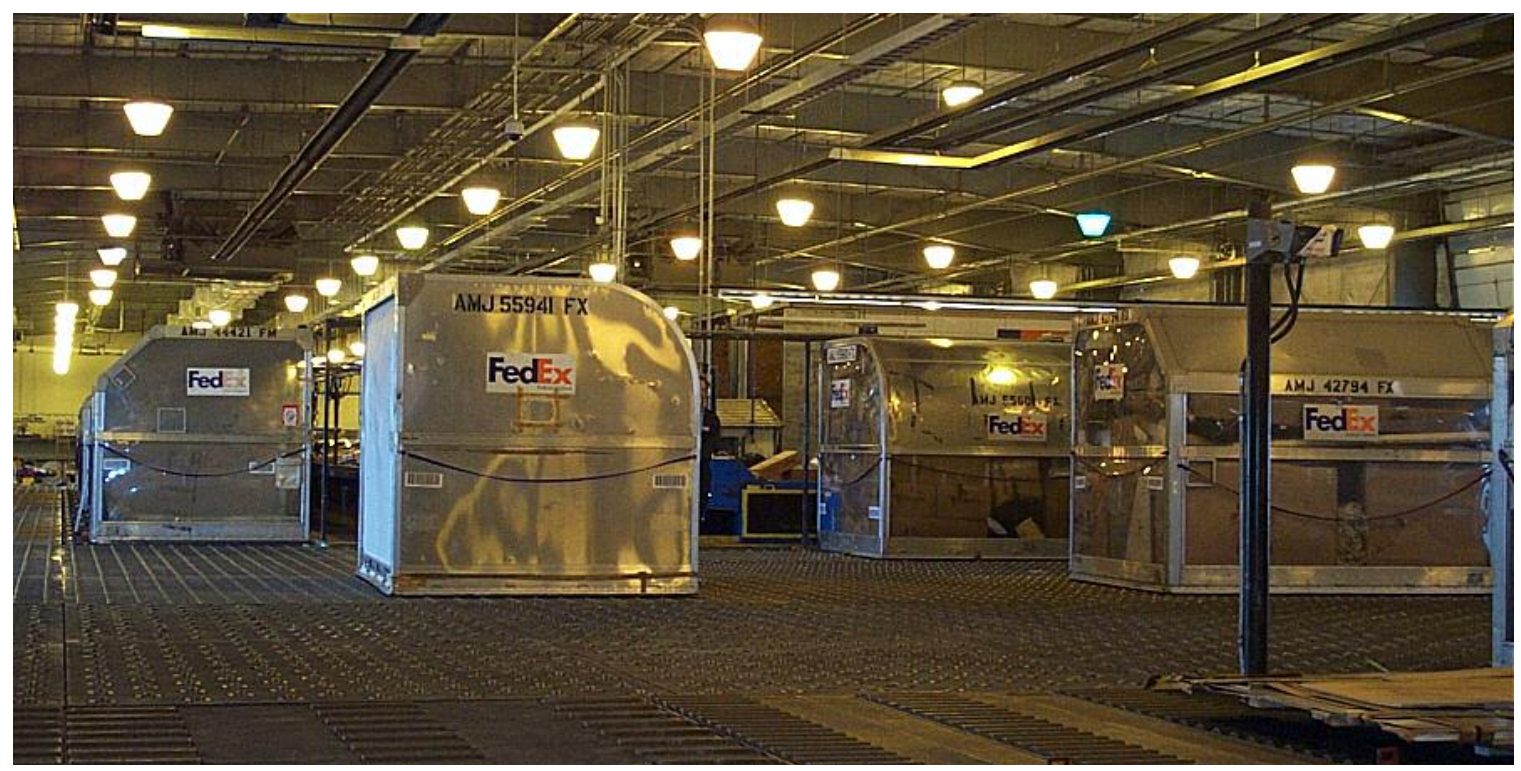

Figure 4. Different Unit Loading Devices (ULDs) used by FedEx. Note the ULDs to the right are full of packages.

\section{Results}

\section{Background Radiation Environment}

Continuous unattended background radiation measurements were acquired over two consecutive weekends, with the HPGe detector the first weekend followed by the ARAM detector the following weekend. Both detectors were located away from the conveyor belt and truck doors in a secured area. The HPGe acquired spectra in 30-minute bins. The ARAM data was summed and rebinned into 30-minute bins for analysis. A plot of gross counts versus time is shown in Figure 5. Analysis of data from the HPGe detector revealed that the background radiation environment did not seem to change significantly with time. The HPGe background radiation measurement varied by approximately $2 \%$ over a 48-hour period. The ARAM data showed significant count rate increases that were not seen in the HPGe data. The ARAM data varied by approximately $20 \%$ over the same 48-hour period the following weekend. In Figure 6, note the increase in background radiation counts for both detectors beginning around 4:30 am, Saturday morning and lasting approximately 2.5 hours. Also note the large increase in background radiation for the ARAM detector around 11 am Sunday morning. The measurement remains high for approximately 3.5 hours. This large increase may be explained by two large blue plastic barrels labeled potassium acetate that at one point during unattended operations were located near the ARAM detector. The potassium acetate generated a detectable radiation environment due to ${ }^{40} \mathrm{~K}$ that was approximately three times the 
radiation background at other locations in the facility. The large blue barrels were not present during the HPGe background measurements the previous weekend.

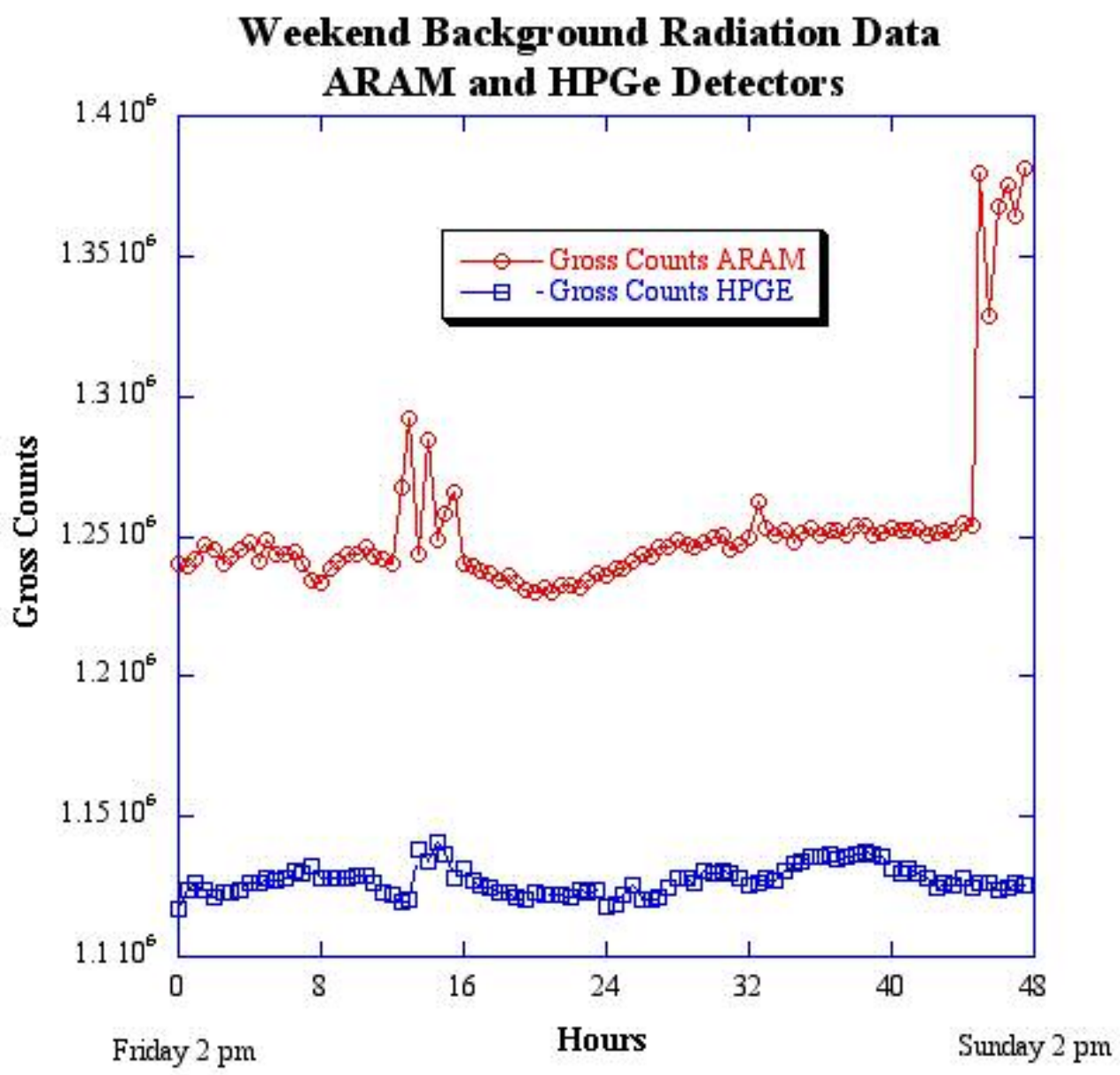

Figure 5. Plot of background radiation gross counts taken with the ARAM detector and the High Purity Germanium detector over 48 hours on consecutive weekends. Data was acquired in 30-minute bins.

\section{Quantifying "nuisance" alarms}

A "nuisance" alarm is defined here as an explainable, legitimate radiation event. The ARAM screened over 24,000 individual packages that passed on the conveyor belt with no radiation events detected. This event rate is not inconsistent with past experiments performed by Sandia National Laboratories (SNL) and Los Alamos National Laboratory (LANL) $[2,3,4]$. The SNL radiation detection experiment performed at the FedEx facility in Anchorage, AK detected 29 radiation events over approximately 20 weeks of continuous operation. Federal Express instrumented the conveyor belt at their Indianapolis Sorting Center with gamma and neutron detection and had only two nuisance alarms in over 9 months of operation [5]. The ARAM in Denver was operational during three evening shifts for a total time of approximately 14.25 hours. In addition, FedEx procedures require all packages considered dangerous goods (including radioactive packages) are handled in the Dangerous Goods area, and do not pass on the conveyor belt. 
The ARAM screened more than 28 trucks as ULDs were unloaded over 4 evening shifts and detected two radiation events. When the ARAM indicated an event had occurred, the secondary handheld detectors were immediately used to locate the radiation emitting regions within the large ULD. Each package was screened using the handhelds in "search" mode as the ULDs were unloaded, and the hot packages were located. Both radiation events that triggered the ARAM were identified as packages containing legitimate medical isotopes, and were labeled appropriately.

The first ARAM event occurred around 20:45 on 25 March 2003. The gross count rate was approximately 5 times background (see Figure 6).

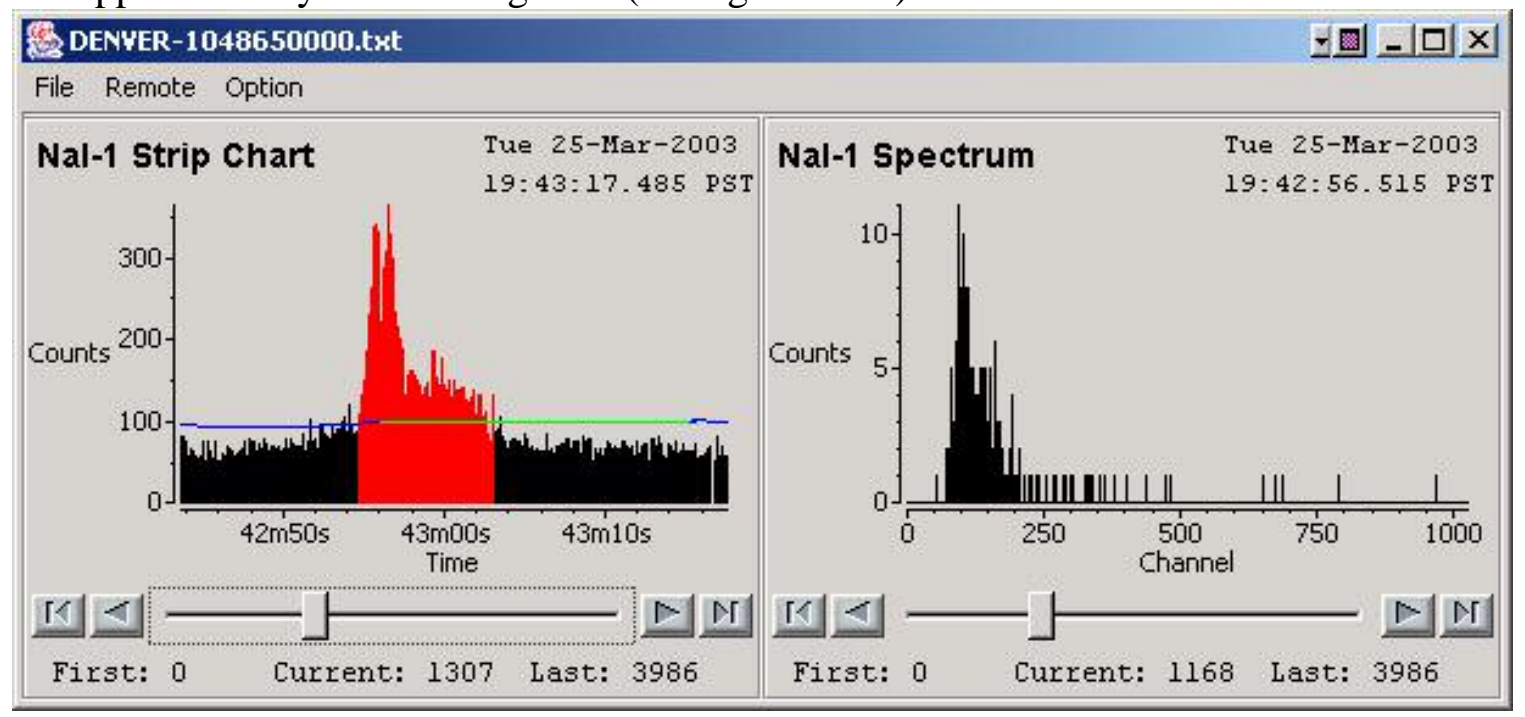

Figure 6. ARAM radiation event on 25 March 2003. The left window displays gross counts and the right window displays spectral data for a single time bin.

The ULD that triggered the event was immediately pulled aside and the three handheld instruments were used to scan the outside of the container in search mode. When the hotter spots were located, the units acquired spectra and attempted to identify the isotope. As the ULD was unloaded, six packages were located and identified as emitting radiation. Each package was labeled as a DOT "excepted package - limited quantity" and they were passed directly to FedEx Dangerous Goods personnel. The SAM 935 and the fieldSpec identified the isotope as ${ }^{131} \mathrm{I}$, while the Exploranium GR-130 was unable to identify. However, upon further analysis, the spectral data from the Exploranium GR130 confirmed the presence of ${ }^{131} \mathrm{I}$. Next, a spectrum was acquired for 300 seconds with the HPGe and the presence of ${ }^{131}$ I was confirmed by a spectroscopist (see Figure 7). 
Package_Northern_scientific_2200

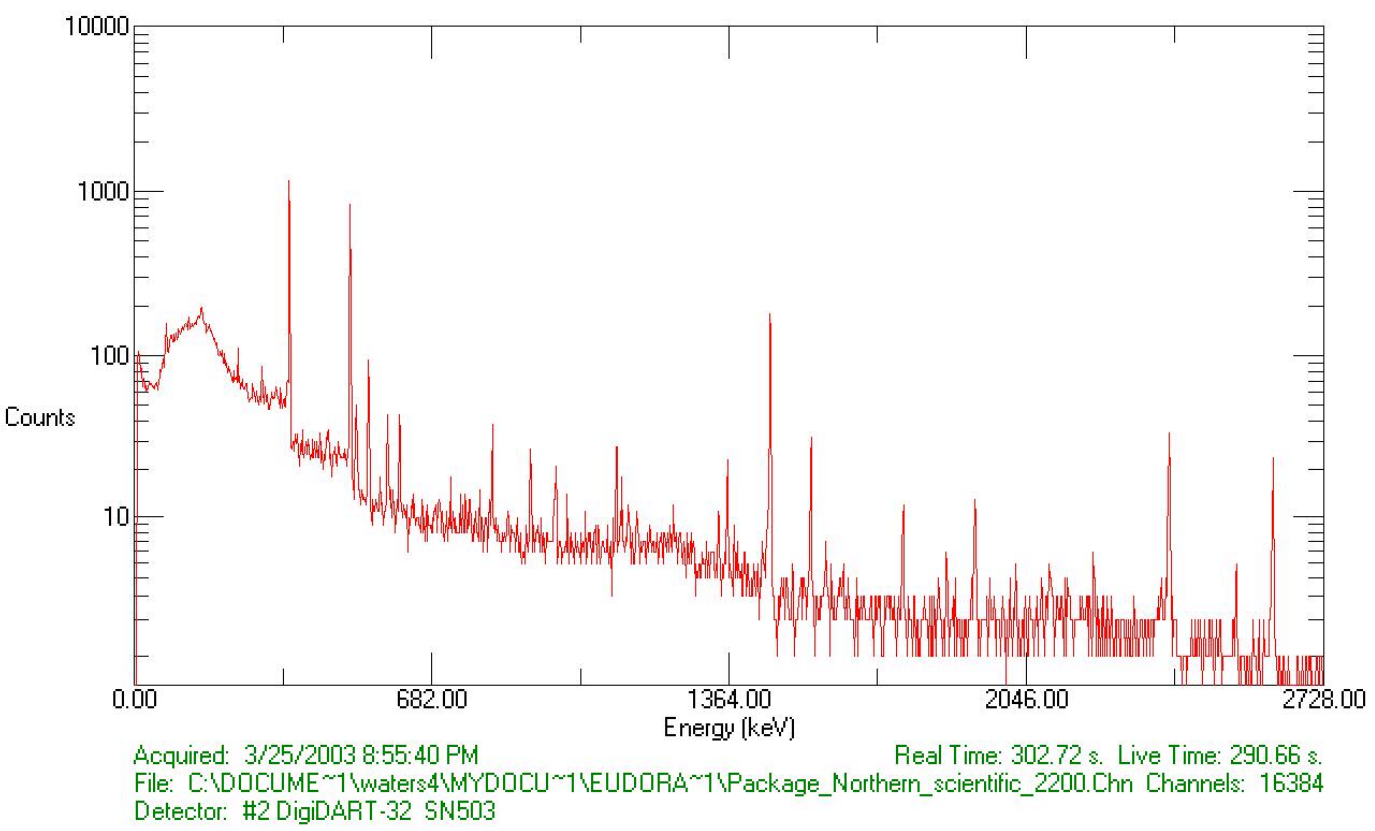

Figure 7. Spectral data acquired with the HPGe for 5 minutes for a package determined to contain ${ }^{131} \mathrm{I}$.

The second ARAM event occurred at 20:37 the following evening and is shown in Figure 8.

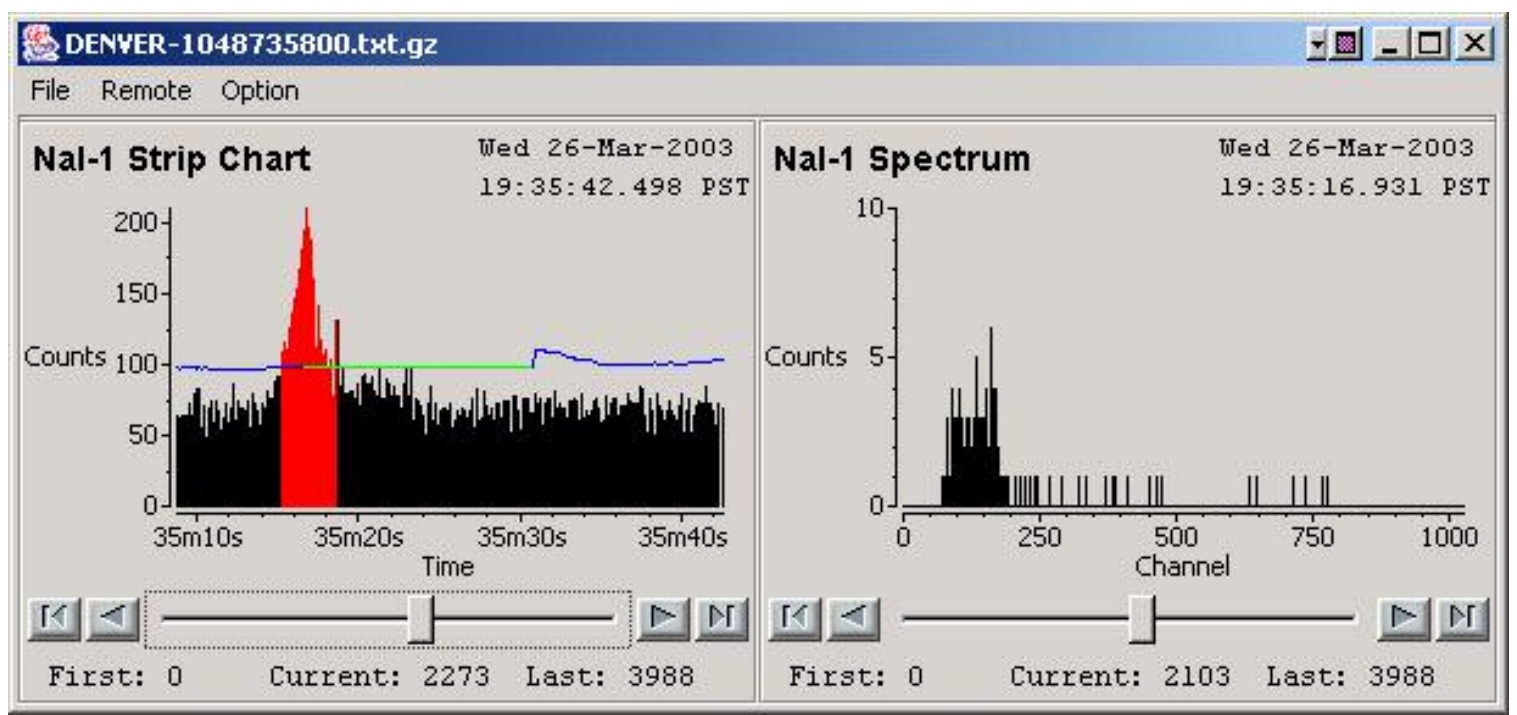

Figure 8. ARAM radiation event on 26 March 2003. 
Again, the ULD was pulled aside and scanned with the handheld instruments. A package emitting radiation was located using the fieldSpec and the Exploranium in search mode. The package was labeled as "Radioactive Class II" and indicated it contained ${ }^{123} \mathrm{I}$. The fieldSpec identified three different isotopes: ${ }^{140} \mathrm{Ba},{ }^{115} \mathrm{Cd}$ and ${ }^{132} \mathrm{I}$. The Exploranium GR130 was unable to identify the isotope. Once again, upon closer examination of the handheld detector data at a later time, the spectral data from the Exploranium as well as the fieldSpec confirmed the presence of ${ }^{123}$ I. Due to logistical problems, we were unable to acquire any spectral data with the SAM 935. The HPGe was used to acquire a spectrum that was immediately analyzed by a spectroscopist. The spectrum acquired is shown in Figure 9. The conclusion was that the package most likely contained ${ }^{123}$ I and was possibly shielded with bismuth, due to the absence of prominent low energy spectral lines and the presence of lines characteristic of ${ }^{214} \mathrm{Bi}$. After analysis, the package was passed directly to the FedEx dangerous goods personnel.

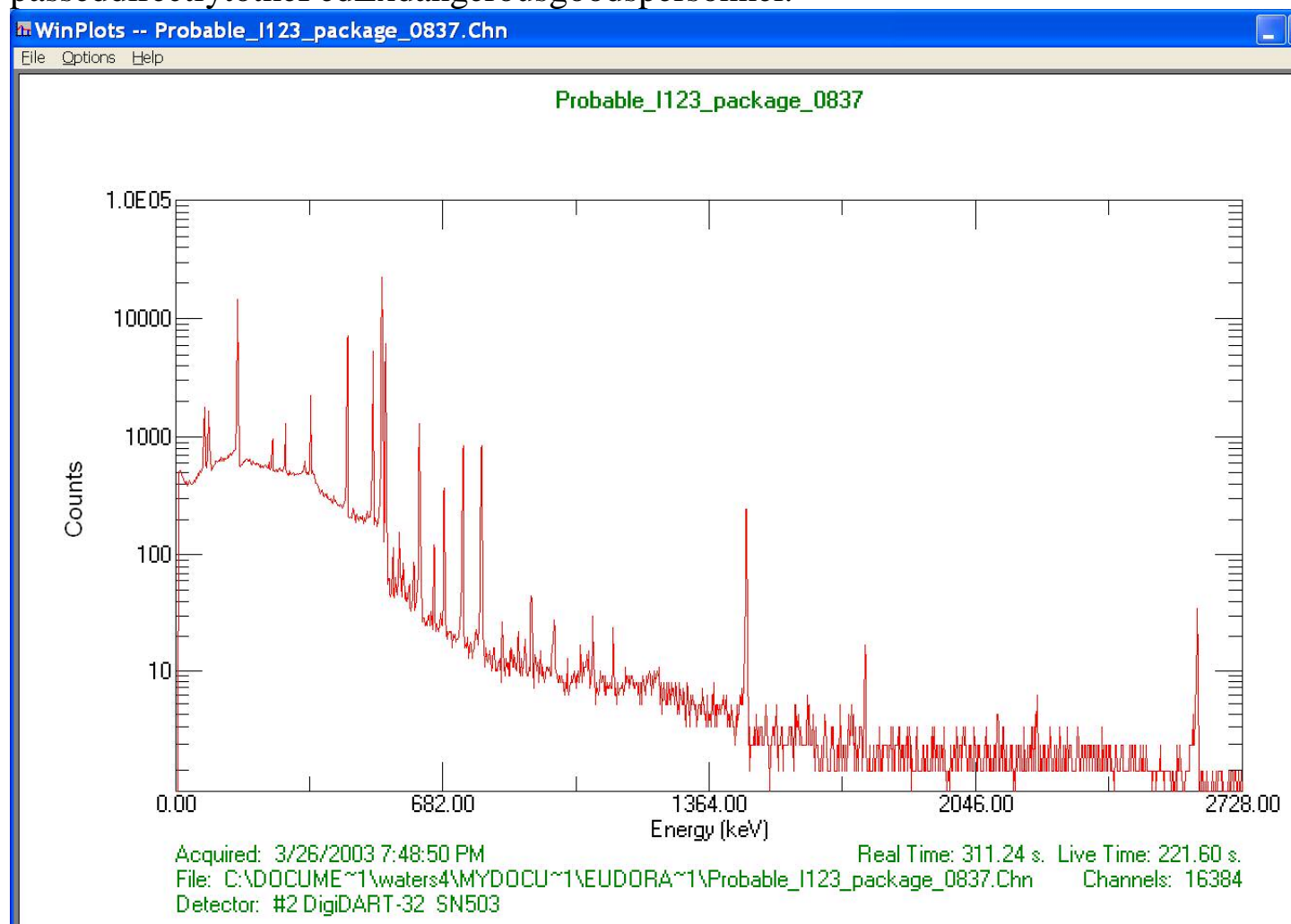

Figure 9. Spectral data for package labeled Rad Class II, ${ }^{123}$ I acquired with the HPGe detector. The absence of prominent lower energy lines characteristic of ${ }^{123}$ I lead us to believe that this isotope was shielded, possibly with bismuth due to the presence of lines characteristic of ${ }^{214} \mathrm{Bi}$.

\section{Evaluation of Detectors in Operational Environment}

ARAM

The ARAM detector worked satisfactorily as a monitor for moving radiation sources in an air cargo facility. We were able to detect small quantities of radiation consistently and in real time. The relatively small size of the ARAM system allowed us to minimize our 
impact on FedEx operations, while the integrated detector design into a ruggedized polyethylene molded shipping case was well suited for the air cargo environment. The version of the ARAM used in this experiment did not have isotope identification capabilities. More recent versions of ARAM have automatic isotope identification software built into the system, along with an automatic trigger for a camera to take a picture when an event occurs. Wireless operation and data transfer would make operations in facilities such as this one easier for the detector operators and even less intrusive to the business.

\section{Handheld detectors}

The handheld detectors were easily portable and in that sense were well suited as secondary, or confirmation detectors. The search modes in both the fieldSpec and the Exploranium were useful, as they both showed a streaming gross count rate as well as employed an audible signal that was easy to understand and interpret quickly. The search mode was used to localize general high radiation areas on the two ULDs that triggered the ARAM. The most striking issuethat was present in all of the handheld units was the importance of having the "correct" isotope library loaded in memory. For example, when the package containing ${ }^{123} \mathrm{I}$ was examined, the spectral data acquired with the fieldSpec and the Exploranium Gr-130 was shown to correctly indicate the presence of isotopes of interest when analyzed back at the lab. However because ${ }^{123}$ I was not loaded into the library of either unit, the identification could not be made at FedEx. One problem we experienced with each of the three handheld units was the difficulty in storing and then retrieving spectral data. In the process of attempting to download data, some spectra were lost.

\section{High Purity Germanium Detector}

This detector was by far the most sensitive, with $140 \%$ efficiency compared to a 3-inch long cylindrical $\mathrm{NaI}(\mathrm{Tl})$ crystal with a 3 -inch diameter. The extremely high resolution of the detector allowed for easier spectral analysis of data. However, analysis of this data requires an experienced spectroscopist; reliable software that automatically identifies isotopes on the fly is not yet available. In addition, the liquid Nitrogen required to cool the HPGe detector to its operating temperature is a safety hazard. A mechanically cooled HPGe detector with built in isotope identification software could be a very powerful tool for secondary identification of anomalous packages in an air cargo environment.

\section{Conclusions and Recommendations}

The strategy used in this field test employing a primary detector with secondary detectors for confirmation worked well in the air cargo environment. Built in isotopic identification software on the primary detector would provide an additional confirmation of identification, and wireless communication would make the experiment less intrusive on business operations. In addition, a high-resolution, high-sensitivity hand carried detector with isotopic identification software such as the recently developed mechanically cooled HPGe detector would further increase the level of confidence in identifications. 
While the background radiation environment did not vary significantly over the first weekend while data was acquired with the HPGe detector, it is very important to be aware of the radiation environment around the detectors. For example, the large increase in background radiation for a period of several hours when data was acquired with the ARAM detector was most likely due to movement of materials that are natural emitters of radiation, such as the barrels full of potassium acetate. Large temperature variations could have affected background measurements, however temperature was not recorded in this experiment.

Over the period of this experiment, two nuisance alarms were detected. To better quantify expected nuisance alarms in an air cargo environment, a much longer test time is required. We detected zero false positives over the course of this experiment.

While the overall approach taken in this experiment was deemed a success, there are several recommendations that can be made with respect to performance of the detectors used.

- Built in isotopic identification on the primary detector would be a benefit.

- The handheld detectors require more robust communication software as well as more intuitive steps to save and analyze collected spectral data.

- Developing and maintaining an appropriate "library" of isotopes is critical. When the downloaded spectral data was analyzed, both the Exploranium GR-130 and the fieldSpec clearly indicated spectral lines from ${ }^{123}$ I, however were not able to identify the isotope because it was not loaded into their libraries.

- An overall strategy for selection of appropriate spectral libraries, levels of rejection of "nuisance" sources, and actions for detection of sources of concern needs to be developed. Software and hardware are mere tools that must be carefully and appropriately applied.

\section{REFERENCES}

1. U.S. General Accounting Office, Aviation Security: Vulnerabilities and Potential Improvements for the Air Cargo System, GAO-03-344 (Washington, D.C.: December 20, 2002).

2. H. L. Scott, D. J. Mitchell, M. W. Johnson, J. A. Bounds, P. M. Steadman, M. P. Dugan, and A. M. Criscuolo, Survey of Radioactive Material at a Port-of-Entry, Sandia National Laboratories, Systems Research Report, January 17, 1997.

3. H. L. Scott, and D. J. Mitchell, Survey of Radioactive Material at a Large International Package Distribution Center, Sandia National Laboratories, Systems Research Report, February 25, 1998.

4. M. W. Johnson, J. A. Bounds, P. A. Steadman, A. Criscuolo and M. P. Dugan, Radionuclides Identified at a U.S. Customs Service Site, Los Alamos National Laboratory, LA-13298-MS, May 1997.

Private communication, Patrick Oppenheimer, Federal Express, Memphis, TN. 